\title{
Development of the Concept vs Prototyping: Implementation of a real scale Water-Energy Integrated System
}

\author{
Ana Rita Silva ${ }^{1,2}$ \\ ${ }^{1}$ ICOVI, EM - \\ Infraestruturas e \\ Concessões da \\ Covilhã \\ Covilhã, Portugal \\ anarita.silva@icovi. \\ $\mathrm{pt}$ \\ ${ }^{2}$ Department of \\ Electromechanical \\ Engineering \\ University of Beira \\ Interior \\ Covilhã, Portugal \\ ana.rita.silva@ubi.pt
}

\author{
Fernando Santos 2,3 \\ ${ }^{2}$ Department of \\ Electromechanical \\ Engineering \\ ${ }^{3}$ Centre for \\ Mechanical and \\ Aerospace Science \\ and Technologies \\ University of Beira \\ Interior \\ Covilhã, Portugal \\ bigares@ubi.pt
}

\author{
António Espírito- \\ Santo $^{2}$ \\ ${ }^{2}$ Department of \\ Electromechanical \\ Engineering \\ University of Beira \\ Interior \\ Covilhã, Portugal \\ aes@ubi.pt
}

\author{
José Páscoa ${ }^{2,3}$ \\ Marques \\ ${ }^{2}$ Department of \\ Electromechanical \\ Engineering \\ ${ }^{3}$ Centre for \\ Mechanical and \\ Aerospace Science \\ and Technologies \\ University of Beira \\ Interior \\ Covilhã, Portugal \\ pascoa@ubi.pt
}

\author{
Cristina Sena Fael ${ }^{4,5}$ \\ ${ }^{4}$ Departament of \\ Civil Engineering \\ and Architecture \\ ${ }^{5}$ Centre of Materials \\ and Building \\ Technologies \\ University of Beira \\ Interior \\ Covilhã, Portugal \\ cmsf@ubi.pt
}

\begin{abstract}
Currently, the concept of smart cities and renewable energy are more consolidated and developed. With each passing day, new technological advances are emerging within both themes. At the same time, the water sector has becoming increasingly demanding in terms of quality and compliance with all existing legislation. That said, it is understandable that both sectors and their management entities come together in search of more sustainable operations that go against the reduction of greenhouse gas emissions, decentralization of energy production and the improvement of efficiency, quality and use of resources. It is important to understand the synergies between water and energy to contradict what has been said so far about the increasing demand for energy, its exponential trend and the negative repercussions on freshwater resources. As such, this article aims to address the interconnection between both sectors to promote alternative scientific and technological improvements to the management and development of Integrated Water-Energy systems, while studying the dynamics between the theoretical development of the problem and the challenges of prototyping of such complex systems in real and urban context.
\end{abstract}

Keywords- Renewable Energy, Energy Maximization, Optimization of Integrated Systems, Prototyping

\section{INTRODUCTION AND BACKGROUND}

The incessant search for a green and more sustainable planet inevitably causes significant efforts and progress by the communities. Although relatively new in Portugal, the theme of the Water-Energy nexus is not recent. In 1994, Peter Gleick published his work on the large-scale life cycle of both resources to quantify the energy intensity in water and vice versa [1]. Although independently developed and regulated, it is known that water needs energy and energy needs water. It is also important to clarify that there will be no strong coherence if all concerns and calls for attention are not incorporated into government policies to improve the management of both sectors. Due to the already long energy crisis, it is necessary to improve the efficiency of the systems and reduce energy consumption, using advanced technological changes and an optimized management strategy, leading to a more sustainable future. In Portugal, in March 2018, energy consumption was for the first time totally produced by renewable energy sources [2]. By improving the efficiency of water and energy use through integrated smart strategies, there are very positive repercussions on savings and impact on the local economy, especially in decentralized cases. In 2014, the United Nations have launched a publication that analyses water demand and availability, energy requirements for water supply and demand for water in energy production. It also suggests that the public policy response to the connection between water and energy requires a hierarchy of actions designed to create an environment conducive to the implementation of the necessary changes for the development of water and energy resources, which focus on the following points [3]: development of coherent policies; legal and institutional structures to promote such coherence; ensuring the reliability of the data and statistics collected for monitoring decision-making; awareness of the problem; support innovation and research in the field of technological development; ensure that funding is available and enable markets and companies to develop in the field. The interdependence between energy and water is nowadays of major importance in all the analyzed literature on the subject, and even then, research and the existence of case studies is very limited. Rapid human growth, centralized industrialization, and improved societal lifestyle are the main factors contributing to the increasement of the demand for resources in most regions of the world. In this context, elaborated within the scope of the 
International Project EdGeWiSe - Energy and Water Systems Integration and Management, this document discusses the importance of the water-energy nexus and integrated management systems as a solution for energy use in municipal water distribution systems, using hydric, solar and wind energy. The paper is structured as follows: Section II presents an overview of integrated water and energy systems. Section III presents the analyzed case study, section IV highlights the energy requirements of the system and the analysis of the data provided. Section V presents the concept development in terms of problem formulation and briefly outlines the challenges and opportunities identified throughout the work. Section VI presents the chosen configuration for the prototype and the last section reveals the main conclusion.

\section{WATER AND ENERGY INTEGRATED SYSTEMS}

Portugal is focused on achieving some of the nexusassociated objectives, as [4]: reducing the consumed energy in water systems by at least $50 \%$; increasing the level of selfsufficiency in terms of energy consumption in wastewater treatment plants; improving the efficiency of operational activities; reducing energy consumption and reactants through the introduction of new technologies, procedures and management concepts; transforming the premises from the places of consumption to places of energy production; increasing wastewater energy recovery; using more renewable energy sources as a substitute for fossil fuels in the urban water cycle without changing the reliability and efficiency of processes and increasing the integration of water services with other sectors in search for regional opportunities (adapting utility to the needs and production locations). These goals are being reviewed by the EdGeWise Project team to find more sustainable solutions to the challenges and barriers of today's water-energy nexus. In Portugal, it is of great importance to change national policies to coordinate the water and energy sectors and to create beneficial synergies to increase the efficiency of resources and to support future generations. Although relatively recent, there is an enormous market potential for nexus in Portugal and it involves both sectors: managing entities (water and energy) seeking additional profit or cost reduction through integrated water and energy solutions, together with intelligent networks, and technology companies that will evolve into a new phase of development to respond to water and energy related problems and constraints. Climate change, pollution and energy insecurity are among the biggest problems of today. There are indeed several measures to be considered which allow the creation of beneficial synergies for water and energy production services such as: innovative studies of integrated water and renewable energy systems, combined energy and desalination plants, combined heat and power plants, the use of alternative water sources for cooling the infrastructure and even the recovery of energy through waste water [5]. From the point of view of sustainable development, the limitations and availability of water for energy production will be critical factors in achieving the SDG (Sustainable Development Goals) of energy. Even if electricity production through wind or photovoltaic system doubled, there would still be a strong need to rely on water sources to achieve universal access to sustainable and viable services and support global economic and industrial growth [6]. Integrated water and energy management systems are also directly related to the concept of smart cities, whether in urban or rural environment, and consequently associated with the Living Lab (LL) concept. According to the Directorate-General for Information Society and Media of the European Communities [7], the concept of a Living Lab is open, and it represents a user-oriented innovation ecosystem based on a partnership between governments and its citizens that allows users to be active in the process development and innovation. It brings users into the creative process of better understanding new and emerging behaviors and patterns; uniting the innovation gap between technological development and the emerging products and services supported by stakeholders, partnerships in business, citizens and governments. Living Labs also assess the socioeconomic implications of new technology solutions, demonstrating the validity of innovative services and business models. It is indeed a sustained fact that the work developed in Living Labs communities has a significant impact on the policy of technological innovation, moving away from linear research activities and towards open innovation. In this sense, the practical case study, in addition to stimulating the union of efforts between public and private entities, overcomes some knowledge gaps and contributes to the scientific advance of the theme, and additionally - after the validation of its installation and operation by the team - will be used in several perspectives of future use, namely: the scope of the optimization of the system in relation to the energy consumed vs energy produced (self-sufficiency); expansion to other potential locations of the city's public water supply network and contribution to further expansion to networks managed by different management entities (inside and outside of Portugal); the use of similar systems in rural context; continuous research throughout the useful life of the equipment, from the monitoring of the ratio of energy produced/energy consumed to the implementation of improvements of applied instrumentation and the system's operation mode; the collection of an extensive database for statistical analysis to be incorporated into research projects that need it.

\section{THE CASE STUDY}

The analyzed case study in this document is within the scope of the EdGeWiSe International Project and refers to the implementation of an integrated water and energy management system in urban context and in real environment in the city of Covilhã, Portugal. Incorporated in the public water supply network, it is intended to take advantage of the gravitational potential of the water flow together with solar and wind renewable energies to meet the energy requirements of the surroundings. The process of developing, scaling and implementing an integrated system as described above, in addition to being iterative, is extremely complex and requires a coordinated and preliminary plan. In addition to identifying all the involved variables, possible scenarios and associated constraints, it is necessary to go through several stages, such as the identification and characterization of the site, pre-feasibility and feasibility studies, detailed design of the integrated energy system and analysis of available funding. Covilhã's supply system has been constantly being maintained and improved, particularly regarding the expansion of the network, installation of tele-management devices and loss control. Recently, and in 
accordance with the EdGeWise Project's objective, the research on the hydroelectric development of the grid for energy production with solar and wind energy is also beginning. The main objective of the implementation of such a system is to optimize the efficiency of energy produced vs energy consumed. Under ideal and constant conditions, the system operates as follows: during the night, hydropower is produced to power the park's public lighting through a PAT (Pump as Turbine) or through a conventional hydraulic turbine (both devices are contemplated in the project for research study and comparison of performance results) and during the day, wind and solar energy are produced to supply a pump that stores water potential in the upper reservoir (to be used at night) and, if possible, an existing recirculation pump of an artificial stream. Therefore, in addition to the choice of the best pipeline combined with the ideal conditions of solar radiation and wind intensity, it is important that it is contained in a semi-closed system conducive to a good quality analysis in order to analyze and monitor multiple variables and scenarios. As such, the Botanical Garden of the City was selected, in which there are two water reservoirs (R02 Superior and R02 Inferior). Fig. 1 shows a schematic drawing of the enclosure.

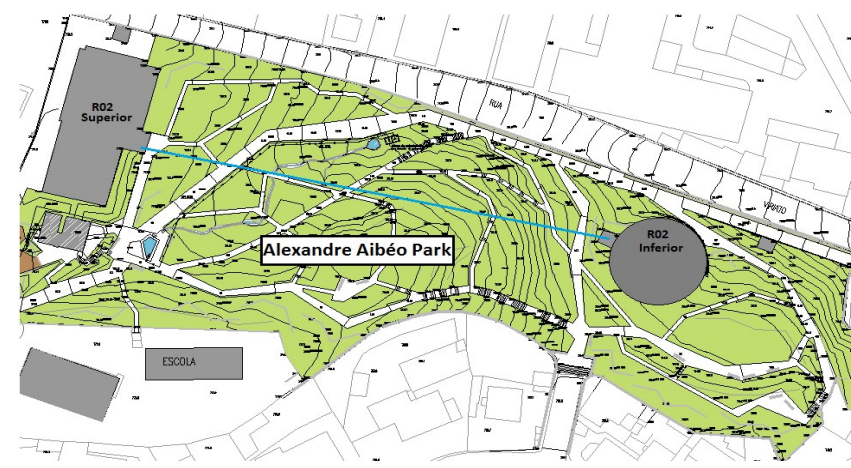

Fig. 1: Schematic drawing of Alexandre Aibéo's Park (Botanical Garden) with the location of both reservoirs (R02 Superior and R02 Inferior).

\section{ENERGY REQUIREMENTS AND AVAILABLE/ANALYZED DATA}

The main energy requirements of the Park are: the consumption of both reservoirs, public lighting, an electric pump to recirculate the water of an artificial stream and an existing building called "Divulgation Center", with a total power of approx. $24 \mathrm{~kW}$. The feasibility study for the development of the theoretical concept and subsequent implementation of the prototype comprises, in a first phase, the hydraulic analysis of the infrastructures of the local public supply network, in which the available flow between both reservoirs and the respective energy potential is determined. Since both water tanks supply a large area and the values represent a typified daily consumption, the study can only benefit in probabilistic terms from occurrence by means of hourly and/or daily average values, depending on the availability of the collected data, which should, as possible, cover a long-time span, in decades, so that a credible and predictable feasibility study can be carried out. Fig. 2 shows the average daily flow duration curves according to the lower reservoir flow output in the years of 2016 and 2017, in which the value of the abscissa axis represents the number of days that a certain flow rate is equal to or exceeded. According to Section III - Case Study, the water energy will supply the street lighting during the night, which, according to the pilot project directive, corresponds to a power of $0.26 \mathrm{~kW}$. Fig. 3 shows the variation in the number of hours between records (green color) and the number of hours at which the power output is higher than the power requirements (orange). The anomalous occurrence registered in January 2017 was due to a failure in the telemanagement device that was temporarily disabled as a consequence of thunderstorm damage. Fig. 4 shows the percentage of time in which the power output is lower than the energy requirements, with the number of hours in the vertical position. It should be noted that in a global way and during the analyzed 21 months, the useful power of the flow is lower than the energetic requirements of public lighting in only $1.56 \%$ of the time.
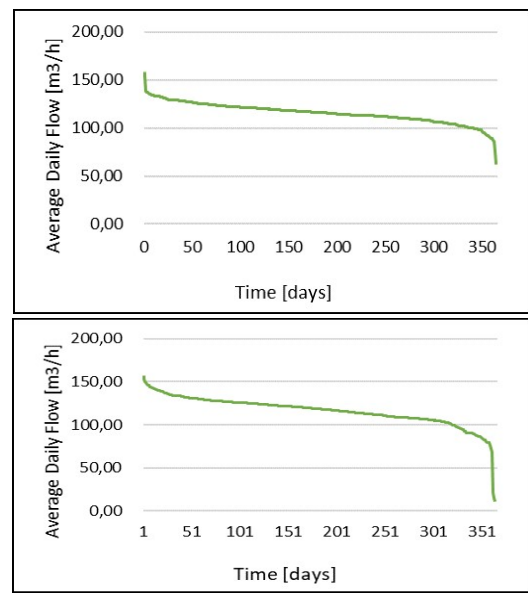

Fig. 2: Average daily flow duration curves for R02 Inferior Reservoir: a) year of 2016; b) year of 2017.

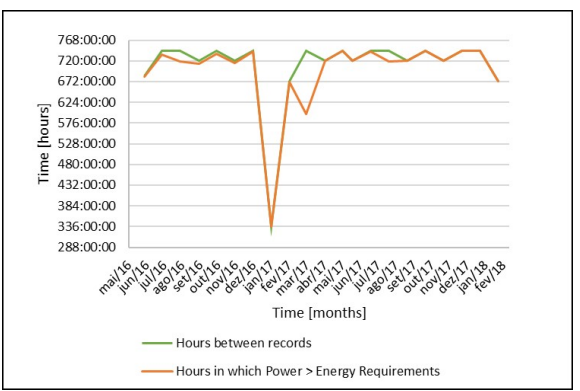

Fig. 3: Number of hours between records vs Number of hours in which Power is higher than energy requirements.

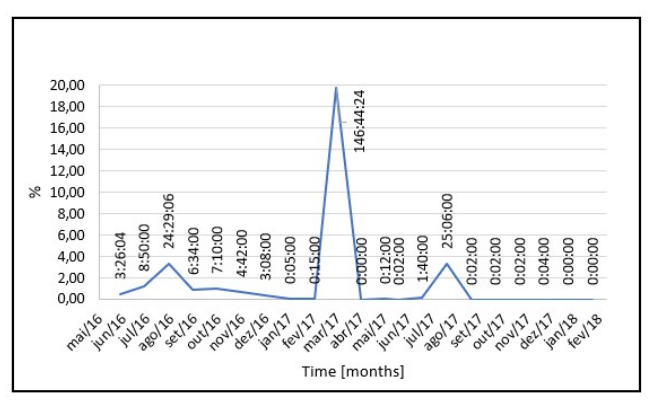

Fig. 4: Percentage of time in which the flow potential power is inferior to the energy requirements (correspondent number of hours in vertical position). 
As for daylight hours, and according to section III, solar and wind energy will supply an electric pump that will store water potential in the upper reservoir and which will have a power of $1 \mathrm{~kW}$ and, if possible, an existing recirculation pump with a power of $2 \mathrm{~kW}$, which makes a total of $3 \mathrm{~kW}$ at night, and in total corresponds to $3 \mathrm{~kW}$ and $28 \mathrm{kWh} /$ day. In this case, two different scenarios are considered: scenario A consists on the operation of the pump for water storage in the upper reservoir for 12 hours and the existing pump for 8 hours (a total of $28 \mathrm{kWh} /$ day) and scenario B corresponds only to the storage pump (12 kWh/day). Fig. 5 shows the monthly average insolation variation between 2016 and 2017 and Fig. 6, the average monthly wind speed in the same period.

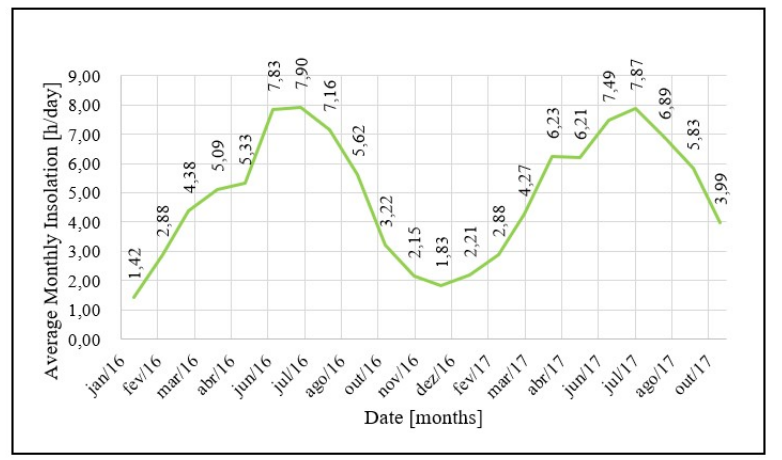

Fig. 5: Average monthly insolation [h/day] in Covilhã, Portugal.

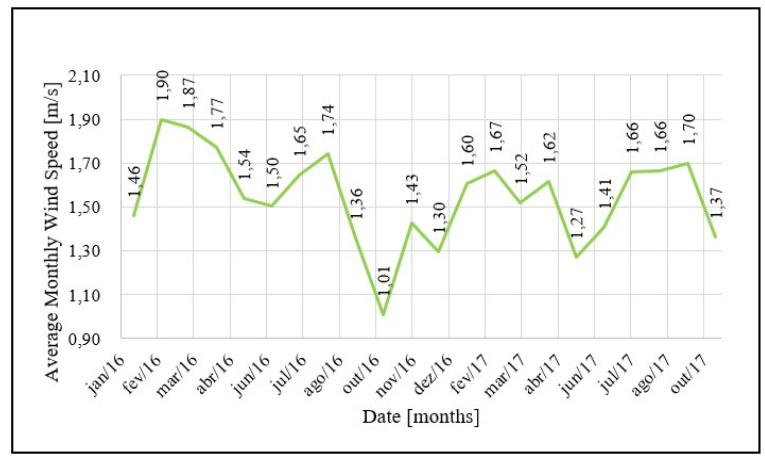

Fig. 6: Average monthly wind speed $[\mathrm{m} / \mathrm{s}]$ in Covilhã, Portugal.

Additionally, Fig. 7 shows the number of $250 \mathrm{~W}$ panels required for scenario A ( $28 \mathrm{kWh} /$ day) and Fig. 8 for scenario B $(12 \mathrm{kWh} /$ day).

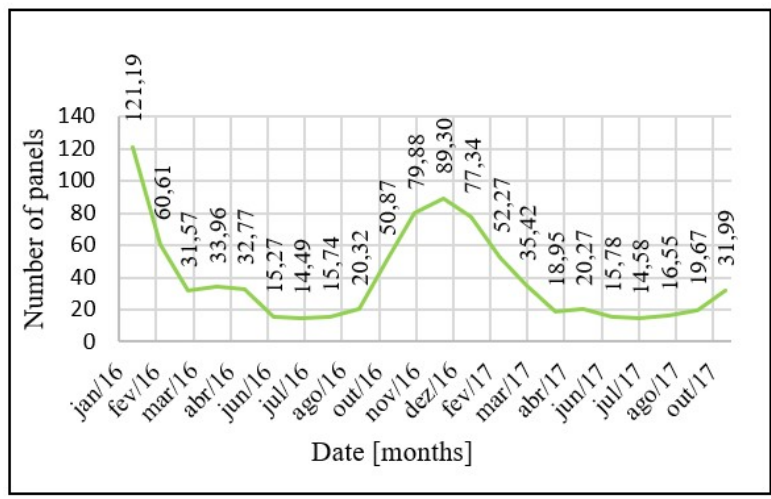

Fig. 7: Number of $250 \mathrm{~W}$ PV panels required for Scenario A.

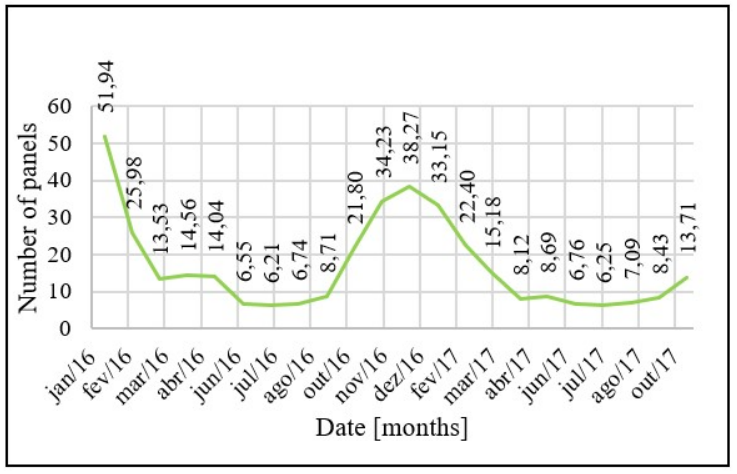

Fig. 8: Number of $250 \mathrm{~W}$ PV panels required for Scenario B.

In case of wind energy, the calculation is slightly different from the solar energy. By applying a generator and multiplier efficiency of $95 \%$ to the mechanical power (in $\mathrm{kWh}$ ) and correlating it with the average wind speed, rotor efficiency $(\mathrm{Cp})$ and specific air weight, the swept area of rotor is obtained. Fig. 9 shows the wind turbine rotor diameter required for scenario $\mathrm{A}$ (28 kWh/day) and Fig. 10 for scenario B (12 kWh/day).

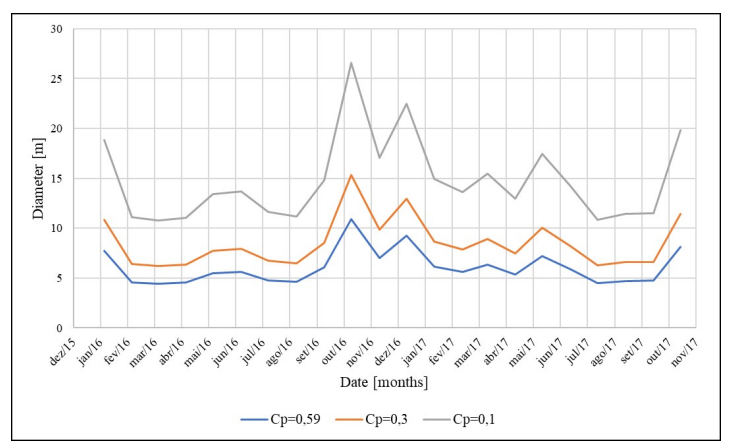

Fig. 9: Rotor Diameter required for Scenario A.

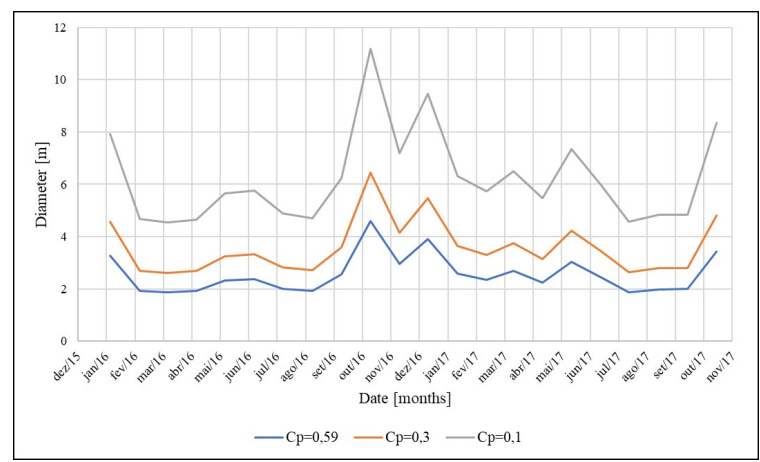

Fig. 10: Rotor Diameter required for Scenario B.

\section{THE DEVELOPMENT OF THE THEORETICAL CONCEPT AND FORMULATION OF THE PROBLEM}

In the scope of the present study, it is intended to implement an integrated water and energy management system in an urban context in a section of a pipeline of the municipal water supply system of Covilhã. To understand the mechanism and interaction between the other intervening factors to be included in the system, it is necessary to cover the subject in a theoretical way. It is an iterative and extremely complex 
process system, and therefore requires a coordinated and organized preliminary plan. To do so, it is necessary to evaluate and study all possible variables, constraints and scenarios to be included in the development of the theoretical concept of the problem. Currently, an optimization model of interaction between different types of energy resources in real environment is being analyzed. In the first phase, an adductor pipeline (125 meters in length and nominal diameter of $200 \mathrm{~mm}$ ) between both reservoirs located in the Park is being analyzed together with the ideal conditions of solar radiation and wind intensity. In developing a theoretical concept of an integrated water and energy system, it is necessary to combat energy needs with various types of renewable energy sources, with the help of conventional energy (if necessary), energy storage and an optimization and management center. The energy requirements of an Integrated Water and Energy System can be met through different scenarios and combinations of renewable energy sources such as wind, sun and water. Any situation that requires more than just a renewable energy source to meet the needs of a given load requires an EMS (Energy Management Strategy) which main objective is to find the balance between the production of renewable energy from unpredictable supply and the fluctuating energy demand [8]. In the present case, the following scenarios and changes in the states of the integrated system are considered: Variation in the production of renewable energies; Variation in water consumption; Variation in energy consumption; Variation of energy and water storage levels in reservoirs; Integrated system layout. The development and implementation of integrated systems require a decision support system that considers all economic, environmental and social factors as well as energy management actions, states and modes of the system so that integration between all reflects in the efficient and optimized management of water and energy systems. To the variables and scenarios mentioned above, and regarding the implementation of the prototype under study, it is important to add the restrictions that limit the application of the concept developed. Such restrictions are at the level of: the place of implementation (the prototype will be installed in the Botanical Garden of the city of Covilhã and in addition it is limited to the park enclosure, it is also easily accessible and subjected to vandalism, so the identification of all the critical points, risky areas and potential slip areas is important); infrastructure impact (due to the weak state of both reservoirs and to neutralize the impact on the existing water system, it was necessary to establish a maximum diameter for the new pipes to be installed); the indispensable outputs of the system (the supply of water to the population that cannot be compromised and the energy consumption associated with the system); the mode of operation (which may change if the preliminary results of the study justify the modification); the current legislation; the available funding; and finally - social and environmental impact.

\section{The Configuration of THE Prototype}

Due to the number of variables and constraints involved in the prototype development, the configuration of the integrated management system to be installed is extremely complex. Of a total of 96 scenarios, 56 are feasible. It was considered relevant to analyze the various scenarios to choose the most appropriate one. These scenarios are a result of the combination between three main categories: 1) the type and quantity of hydric devices to install (pump/PAT + conventional hydric turbine), 2) the number of pipelines to install (one, one and a bypass, or two pipelines) and 3 ) the type of electric devices in the park to be supplied (both water tanks, public illumination, the existing pump, the existing building and the different combinations between all of the devices). In other words, among the analyzed sets of variables, it was considered: the hydroelectric power generation equipment, the piping to be installed and the number of devices to be supplied. For example, a scenario that would be impossible to test would be for the prototype to supply energy for all the energy requirements at the same time (existing building and pump, public lighting and both reservoirs) - due to the available financial support. Another example of a non-feasible scenario would be the installation of two new pipelines and one pump that would also be functioning as a PAT - it would not make sense for a new pipeline to be installed without hydraulic equipment. The first objective is to determine the water net head, the available flow, the orientation of solar radiation and the intensity and direction of the wind, whose dependence stochastic nature will influence the whole process of development and design of the prototype. Fig. 11 shows the sketch of the chosen scenario, including the installation of two new pipelines, which from the point of view of research, is the solution that allows to study more scenarios and combinations of interest to the object of study. For example, if in any circumstance, the upper reservoir would be short on water, it would be necessary to urgently pump water from the bottom reservoir, even if the PAT/hydraulic turbine is functioning to produce energy and to supply the needed public lighting at a certain moment.

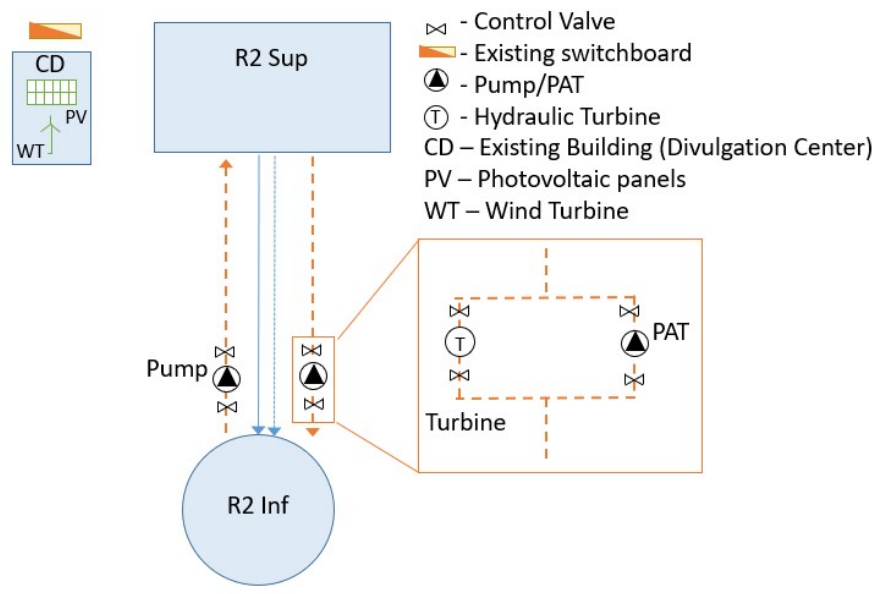

Fig. 11: Integrated Energy and Water system to implement in Alexandre Aibéo Park (Botanical Garden).

The chosen solution is defined by an installation of two new pipelines. The pipe that will have the flow direction opposite to the gravitational flow, will be operated by a pump that will be installed in normal mode to store water potential in the upper reservoir, which under ideal conditions, will work in daytime. The second pipe will be complemented with a PAT (reverseacting pump) and a conventional hydraulic turbine to compare the results between both devices. The main objectives of the team responsible for the installation and operation of the prototype are: Implement, supervise, correct and manage all the 
involved variables, scenarios and restrictions throughout the execution and analysis of the project; optimize the integrated system installed in the park in terms of energy and water; analyze the technical aspects and specifications of the applied instrumentation; optimization of PAT production The implementation of the described system in the document, from the ideal point of view of the mode of operation, requires realtime information complemented with forecast model data. Such information enables the intelligent management of the integrated energy and water distribution system so that it reaches its maximum potential in terms of production and consumption. In addition, the water level within the reservoirs must be known in real time to efficiently store the energy produced by water as a potential of gravitational energy. Since the main function of a water distribution network is to provide water to final consumers, any disruption in the service and minimized impact will be avoided (the impact must stay neutral at all costs). The continuity of service cannot be compromised. Simultaneously, a wireless sensor network (WSN) will be installed and it will meet all safety requirements. Connecting a battery-powered WSN will require a periodic maintenance service to replace them, which is impractical if the number of sensor nodes is large and hardly accessible. By collecting energy from the integrated system to be installed or the surrounding environment (for example, from vibrations, thermal gradients, daylight radiation or microbial fuel cells), the WSN becomes energetically independent.

\section{CONCLUSION}

This paper aims to emphasize the importance of the development of the theoretical concept in the implementation of a prototype on real scale and in an urban context. This is a practical case study within ERANETMED transnational cooperation, dedicated to the coordination of research and innovation for societal challenges in the Mediterranean region. The concept of water-energy nexus was approached and applied in the development of the described prototype installation project, which configuration and optimization were applied according to all associated constraints. The study was initiated involving three variables, the production of electricity through mini-hydro, mini-solar and mini-wind, and the simultaneous consumption in stochastic environment. The work will be further developed using a mathematical formulation of the problem, simulation systems and the implementation of a wireless sensor network (WSN) that will collect information from the pilot to be installed for later determination of the optimum operating point. With the system described, it is possible to analyze the environmental and economic impacts of the system while simultaneously promoting efficiency and reliability.

\section{ACKNOWLEDGMENT}

The authors would like to thank the Foundation for Science and Technology (FCT) for the financial support (Grant ERANETMED/0004/2014), at the initiative of the ERANETMED member states, the associated countries, the Mediterranean partner countries (Project ID eranetmed_nexus14- 044), the Department of Electromechanical Engineering and C-MAST - Center for Aerospace Science and Technologies, of University of Beira Interior.

\section{REFERENCES}

[1] Water in the West, Stanford University, Water Energy Nexus: A Literature Review, Stanford, 2013.

[2] APREN - The Portuguese Renewable Energy Association, [Online]. Available: www.apren.pt. [Accessed on 12 April 2018].

[3] UNESCO, "The United Nations World Water Development Report 2014: Water and Energy"," United Nations World Water Assessment Programme, Paris, 2014.

[4] A. Serra, "Nexus Água - Energia," 16 November 2012. [Online]. Available: http:/www.ppa.pt/wp-content/uploads/2012/12/AlexandraSerra.pdf. [Accessed on 12 September 2017].

[5] P. S. A. Espírito-Santo, "Harvesting Energy from Microbial Fuel Cells: Powering Wireless Sensor Networks Operating in Wastewater Treatment Plants," Biologically-Ispired Energy Harvesting through Wireless Sensor Technologies, IGI Global - Disseminator of Knowledge, pp. 121-171, 2016.

[6] UNESCO, "Water for a Sustainable World," Paris, 2015.

[7] Commission of the European Communities, Living Labs for User-driven Open Innovation - An overview of the living labs ethodology, activities and achievements, Vols. \%1 de \%2ISBN 978-92-79-10358-2/DOI 10.2759/34481, Luxembourg: Information Society and Media Directorate-General, 2009.

[8] D. Assimacopoulos, "Energy management in water systems Deliverable 4.3," EdGeWiSe Project, Greece, 2017. 